PROCEEDINGS OF THE

AMERICAN MATHEMATICAL SOCIETY

Volume 137, Number 5, May 2009, Pages 1807-1811

S 0002-9939(08)09799-2

Article electronically published on December 23, 2008

\title{
ON THE TOPOLOGY OF MANIFOLDS WITH POSITIVE ISOTROPIC CURVATURE
}

\author{
SIDDARTHA GADGIL AND HARISH SESHADRI
}

(Communicated by Jon G. Wolfson)

\begin{abstract}
We show that a closed orientable Riemannian $n$-manifold, $n \geq 5$, with positive isotropic curvature and free fundamental group is homeomorphic to the connected sum of copies of $S^{n-1} \times S^{1}$.
\end{abstract}

\section{INTRODUCTION}

Let $(M, g)$ be a closed, orientable, Riemannian manifold with positive isotropic curvature. By [9], if $M$ is simply connected, then $M$ is homeomorphic to a sphere of the same dimension. We shall generalise this to the case when the fundamental group of $M$ is a free group.

Theorem 1.1. Let $M$ be a closed, orientable Riemannian n-manifold with positive isotropic curvature. Suppose that $\pi_{1}(M)$ is a free group on $k$ generators. Then, if $n \neq 4$ or $k=1$ (i.e. $\pi_{1}(M)=\mathbb{Z}$ ), $M$ is homeomorphic to the connected sum of $k$ copies of $S^{n-1} \times S^{1}$.

We note that a conjecture of M. Gromov (4], Section 3 (b)) and A. Fraser [2, based on the work of Micallef-Wang 8, states that any compact manifold with positive isotropic curvature has a finite cover satisfying our hypothesis.

Conjecture 1 (M. Gromov, A. Fraser). $\pi_{1}(M)$ is virtually free; i.e., it is a finite extension of a free group.

It is known by the work of A. Fraser [2] and A. Fraser and J. Wolfson 3 that $\pi_{1}(M)$ does not contain any subgroup isomorphic to the fundamental group of a closed surface of genus at least one.

Our starting point is the following fundamental result of M. Micallef and J. Moore [9].

Theorem 1.2 (M. Micallef, J. Moore). Suppose $M$ is a closed manifold with positive isotropic curvature. Then $\pi_{i}(M)=0$ for $2 \leq i \leq \frac{n}{2}$.

It is clear that the following purely topological result, together with the MicallefMoore theorem, implies Theorem 1.1.

Theorem 1.3. Let $M$ be a smooth, orientable, closed n-manifold such that $\pi_{1}(M)$ is a free group on $k$ generators and $\pi_{i}(M)=0$ for $2 \leq i \leq \frac{n}{2}$. If $n \neq 4$ or $k=1$, then $M$ is homeomorphic to the connected sum of $k$ copies of $S^{n-1} \times S^{1}$.

Received by the editors July 29, 2008.

2000 Mathematics Subject Classification. Primary 53C21.

(C)2008 American Mathematical Society Reverts to public domain 28 years from publication 
Henceforth let $M$ be a smooth, orientable, closed $n$-manifold such that $\pi_{1}(M)$ is a free group on $k$ generators and $\pi_{i}(M)=0$ for $2 \leq i \leq \frac{n}{2}$. We assume throughout that all manifolds we consider are orientable.

Let $\widetilde{M}$ be the universal cover of $M$. Hence $\pi_{1}(\widetilde{M})$ is trivial and so is $\pi_{i}(\widetilde{M})=$ $\pi_{i}(M)$ for $2 \leq i \leq \frac{n}{2}$. We shall show that the homology of $\widetilde{M}$ is isomorphic as $\pi_{1}(M)$-modules to that of the connected sum of $k$ copies of $S^{n-1} \times S^{1}$. We then show that $M$ is homotopy equivalent to the connected sum of $k$ copies of $S^{n-1} \times S^{1}$ using theorems of Whitehead. Finally, recent results of Kreck and Lück allow us to conclude the result.

\section{The homology of $\widetilde{M}$}

Let $X$ denote the wedge $\bigvee_{j=1}^{k} S^{1}$ of $k$ circles and let $x$ denote the common point on the circles. Choose and fix an isomorphism $\varphi$ from $\pi_{1}(M, p)$ to $\pi_{1}(X, x)$ for some basepoint $p \in M$. We shall use this identification throughout. Denote $\pi_{1}(M, p)=\pi_{1}(X, x)$ by $\pi$.

As $X$ is an Eilenberg-Mac Lane space, there is a map $f:(M, p) \rightarrow(X, z)$ inducing $\varphi$ on fundamental groups and a map $s:(X, z) \rightarrow(M, p)$ so that $f \circ s: X \rightarrow X$ is homotopic to the identity.

We deduce the homology of $\widetilde{M}$ using the Hurewicz Theorem and Poincaré duality.

Lemma 2.1. For $1 \leq i \leq n / 2, H_{i}(\widetilde{M}, \mathbb{Z})=0$.

Proof. As $\widetilde{M}$ is simply connected and $\pi_{i}(\widetilde{M})=\pi_{i}(M)=0$ for $1<i \leq n / 2$ (by hypothesis), by the Hurewicz theorem, $H_{i}(\widetilde{M}, \mathbb{Z})=0$ for $1 \leq i \leq n / 2$.

We deduce the homology in dimensions above $n / 2$ using Poincaré duality for $M$ with coefficients in the module $\mathbb{Z}[\pi]$, namely

$$
H_{n-i}(M, \mathbb{Z}[\pi])=H^{i}(M, \mathbb{Z}[\pi]) .
$$

Recall that $H_{k}(M, \mathbb{Z}[\pi])=H_{k}(\widetilde{M}, \mathbb{Z})$ and the group $H^{i}(M, \mathbb{Z}[\pi])$ is the cohomology with compact support $H_{c}^{i}(\widetilde{M}, \mathbb{Z})$. Hence Poincaré duality with coefficients in $\mathbb{Z}[\pi]$ is the same as Poincaré duality for a non-compact manifold relating homology to cohomology with compact support.

To apply Poincaré duality, we need the following lemma.

Lemma 2.2. For $1 \leq i \leq n / 2$, the map $s:(X, z) \rightarrow(M, p)$ induces isomorphisms of modules with $s_{*}: H^{i}(M ; \mathbb{Z}[\pi]) \rightarrow H^{i}(X ; \mathbb{Z}[\pi])$.

Proof. As the map $s$ induces an isomorphism on homotopy groups in dimensions at most $n / 2$, it induces isomorphisms on the cohomology groups with twisted coefficients. Specifically, we can add cells of dimensions $k \geq n / 2+2$ to $M$ to obtain an Eilenberg-MacLane space $\bar{M}$ for the group $\pi$, which is thus homotopy equivalent to $X$. For $i \leq n / 2$ and any $\mathbb{Z}[\pi]$-module $A$, it follows that

$$
H_{i}(M, A)=H_{i}(\bar{M}, A)=H_{i}(X, A),
$$

where the first equality follows as the cells added to $M$ to obtain $\bar{M}$ are of dimension at least $n / 2+2$ and the second as the spaces are homotopy equivalent.

By applying Poincaré duality, we obtain the following result. 
Lemma 2.3. Let $M$ be a smooth, orientable, closed $n$-manifold such that $\pi_{1}(M)$ is a free group on $k$ generators and $\pi_{i}(M)=0$ for $2 \leq i \leq \frac{n}{2}$. Then, for the universal cover $\widetilde{M}$ of $M$,

(1) $H_{i}(\widetilde{M}, \mathbb{Z})=0$ for $1 \leq i<n-1$.

(2) We have an isomorphism $H_{n-1}(\widetilde{M}, \mathbb{Z})=H_{c}^{1}(\widetilde{X}, \mathbb{Z})$, where $\tilde{X}$ is the universal cover of $X$, determined by the isomorphisms $s_{*}: \pi_{1}(X, z) \rightarrow \pi_{1}(M, p)$ on fundamental groups.

Proof. The statements follow from Lemmas 2.1 and 2.2 by using $H_{*}(\widetilde{M}, \mathbb{Z})=$ $H_{*}(M, \mathbb{Z}[\pi])$.

\section{3. Номотору тYре}

We now show that $M$ is homotopy equivalent to the connected sum $Y$ of $k$ copies of $S^{n-1} \times S^{1}$. Our first step is to construct a map $g: Y \rightarrow M$. We shall then show that it is a homotopy equivalence.

Note that $Y$ has the structure of a CW-complex obtained as follows. The 1skeleton of $Y$ is the wedge $X$ of $k$ circles. Let $\alpha_{i}$ denote the $i$ th circle with a fixed orientation.

We attach $k(n-1)$-cells $D_{j}$, with the $j$ th attaching map mapping $\partial D^{n-1}$ to the midpoint $x_{j}$ of the $j$ th circle. Finally, we attach a single $n$-cell $\Delta$.

We associate to $D_{j}$ an element $A_{j} \in \pi_{n-1}(Y, x)$. Namely, as the attaching map is constant, the $j$ th $(n-1)$-cell gives an element $B_{j} \in \pi_{n-1}\left(Y, x_{j}\right)$. We consider the subarc $\beta_{j}$ of $\alpha_{j}$ joining $z_{j}$ to $x$ in the negative direction and let $A_{j}$ be obtained from $B_{j}$ by the change of basepoint isomorphism using $\beta_{j}$.

Note that if we instead chose the arc joining $z_{j}$ to $x$ in the positive direction, then the resulting element is $-\alpha_{j} \cdot A_{j}$. By the construction of $Y$, it follows that the attaching map of the $(n-1)$-cell represents the element

$$
\partial \Delta=\Sigma_{j}\left(A_{j}-\alpha_{j} \cdot A_{j}\right)
$$

in $\pi_{n-1}(Y)$ regarded as a module over $\pi_{1}(Y)$. This can be seen for instance by using Poincaré duality.

We now construct the map $g: Y \rightarrow M$. Recall that we have a map $s:(X, z) \rightarrow$ $(M, p)$ inducing the isomorphism $\varphi^{-1}$ on fundamental groups. We define $g$ on the 1-skeleton $X$ of $Y$ by $\left.g\right|_{X}=s$. We henceforth identify the fundamental groups of $Y$ and $M$ using the isomorphism $\varphi$, i.e., $\pi_{1}(Y, z)$ is identified with $\pi$.

We next extend $g$ to the $n$-cell of $Y$ as follows. By the Hurewicz theorem and Lemma 2.3 we have isomorphisms of $\pi$-modules $\pi_{n-1}(M, p)=H_{n-1}(\widetilde{M}, \mathbb{Z})$ and $\pi_{n-1}(Y, z)=H_{n-1}(\widetilde{M}, \mathbb{Z})$. By Lemma 2.3, each of these modules is isomorphic to $H_{c}^{1}(\widetilde{X}, \mathbb{Z})$ with the isomorphisms determined by the identifications of the fundamental groups.

Under the above isomorphisms the elements $A_{j}$ correspond to elements $A_{j}^{\prime}$ in $\pi_{n-1}(M, p)$. Consider the element $B_{j}^{\prime}$ of $\pi_{n-1}\left(M, g\left(z_{j}\right)\right)$ obtained from $A_{j}^{\prime}$ by the basechange map using the arc $f\left(\beta_{j}\right)$. We define the map $g$ on $D_{j}$ by extending the constant map on its boundary to be a representative of $B_{j}$.

As the $\pi$-modules $\pi_{n-1}(M, p)$ and $\pi_{n-1}(Y, z)$ are isomorphic, the image $g$ of $\partial \Delta$ is homotopically trivial. Hence we can extend the map $g$ across the cell $\Delta$.

Lemma 3.1. The map $g: Y \rightarrow M$ is a homotopy equivalence. 
Proof. Let $G: \widetilde{Y} \rightarrow \widetilde{M}$ be the induced map on the universal covers. By Lemma 2.3 applied to $M$ and $Y$, we see that $H_{p}(\widetilde{Y})=H_{p}(\widetilde{M})=0$ for $0<p \neq n-1$ and $G$ induces an isomorphism on $H_{n-1}$. Thus the map $G$ is a homology equivalence. By a theorem of Whitehead [10, a homology equivalence between simply connected CW-complexes is a homotopy equivalence.

It follows that $G$ induces isomorphisms $G_{*}: \pi_{k}(\widetilde{Y}) \rightarrow \pi_{k}(\widetilde{M})$ for $k>1$. As covering maps induce isomorphims on higher homotopy groups, and $g$ induces an isomorphism on $\pi_{1}$, it follows that $g$ is a weak homotopy equivalence, hence a homotopy equivalence (see [5]).

\section{Proof of Theorem 1.3}

The rest of the proof of Theorem 1.3 is based on results of Kreck-Lück [7]. In [7, the authors define a manifold $N$ to be a Borel manifold if any manifold homotopy equivalent to $N$ is homeomorphic to $N$. We have shown that a manifold $M$ satisfying the hypothesis of Theorem 1.3 is homotopy equivalent to the connected sum $Y$ of $k$ copies of $S^{n-1} \times S^{1}$. Hence it suffices to observe that $Y$ is Borel.

By Theorem 0.13(b) of [7], the manifold $S^{n-1} \times S^{1}$ is Borel for $n \geq 4$. This completes the proof in the case when $\pi_{1}(M)=\mathbb{Z}$. Further, if $n \geq 5$, then Theorem 0.9 of [7] says that the connected sum of Borel manifolds is Borel; hence $Y$ is Borel. This concludes the proof for $\pi_{1}(M)$ a free group and $n \geq 5$.

Finally, in the case when $n=3$ by the Knesser conjecture (proved by Stallings) the manifold $M$ is a connected sum of manifolds whose fundamental group is $\mathbb{Z}$. As $M$ is orientable, it follows that if $M$ is expressed as a connected sum of prime manifolds (such a decomposition exists and is unique by the Knesser-Milnor theorem), then each prime component is either $S^{2} \times S^{1}$ or a homotopy sphere. By the Poincaré conjecture (Perelman's theorem), every homotopy 3-sphere is homeomorphic to a sphere. It follows that $M$ is the connected sum of $k$ copies of $S^{2} \times S^{1}$.

\section{ACKNOWLEDGMENT}

We thank the referees for helpful comments and for suggesting a simplification of our proof.

\section{REFERENCES}

1. K. S. Brown, Cohomology of groups, Graduate Texts in Mathematics 87, Springer-Verlag, New York, 1994. MR1324339 (96a:20072)

2. A. M. Fraser, Fundamental groups of manifolds with positive isotropic curvature, Ann. of Math. (2) 158 (2003), no. 1, 345-354. MR1999925 (2004j:53050)

3. A. M. Fraser, J. Wolfson, The fundamental group of manifolds of positive isotropic curvature and surface groups, Duke Math. J. 133 (2006), no. 2, 325-334. MR2225695 (2007h:53050)

4. M. Gromov, Positive curvature, macroscopic dimension, spectral gaps and higher signatures, Functional analysis on the eve of the 21st century, Vol. II (New Brunswick, NJ, 1993), 1-213, Progr. Math., 132, Birkhäuser Boston, Boston, MA, 1996. MR.1389019 (98d:53052)

5. A. Hatcher, Algebraic topology, Cambridge University Press, Cambridge, 2002. MR 1867354 (2002k:55001)

6. H. Hopf, Fundamentalgruppe und zweite Bettische Gruppe, Comment. Math. Helv. 14 (1942), 257-309. MR0006510(3:316e)

7. M. Kreck and W. Lück, Topological rigidity for non-aspherical manifolds, to appear in Quarterly Journal of Pure and Applied Mathematics.

8. M. J. Micallef, M. Y. Wang, Metrics with nonnegative isotropic curvature, Duke Math. J. 72 (1993), no. 3, 649-672. MR1253619 (94k:53052) 
9. M. J. Micallef, J. D. Moore, Minimal two-spheres and the topology of manifolds with positive curvature on totally isotropic two-planes, Ann. of Math. (2) 127 (1988), no. 1, 199-227. MR 924677 (89e:53088)

10. J. H. C. Whitehead, On simply connected, 4-dimensional polyhedra. Comment. Math. Helv. 22 (1949), 48-92. MR0029171 (10:559d)

Department of Mathematics, Indian Institute of Science, Bangalore-560012, India

E-mail address: gadgil@math.iisc.ernet.in

Department of Mathematics, Indian Institute of Science, Bangalore-560012, India

E-mail address: harish@math.iisc.ernet.in 